NAFLD

\title{
Ketogenesis could be a determinant of steatohepatitis
}

A new study published in The Journal of Clinical Investigation has linked impaired ketogenesis (the production of alternative energy from fatty acid metabolism) to altered hepatic glucose and lipid metabolism. Mice with ketogenic insufficiency had increased de novo lipogenesis (DNL) and gluconeogenesis, and a high-fat diet (HFD) induced histological features of NASH in these animals.

The role of ketogenesis in diet-induced steatohepatitis has not been well characterized. "Classically, ketogenesis is a process that is only induced in the liver during starvation, decompensated type I diabetes, or adherence to Atkins-style diets," explains corresponding author Peter Crawford. "Our studies were designed to show that ketogenesis is important to consider in the normal-fed state and may be significant in obesity."

A key enzyme

involved in ketogenesis is 3-hydroxymethylglutaryl-CoA synthase 2 (HMGCS2). This enzyme was knocked down by administering antisense oligonucleotides causing ketogenic insufficiency in mice. The effect of subsequent metabolic adaptations on the pathogenesis of steatohepatitis was studied in the context of normal (low-fat) diet and over-nutrition.

HMGCS2-knockdown mice on a low-fat diet (LFD) had mild hyperglycaemia, increased hepatic gluconeogenesis from pyruvate and a metabolic profile indicative of enhanced DNL compared with control mice injected with a scrambled antisense oligonucleotide. Liver tissue from HMGCS2-knockdown mice on a HFD had a NASHlike phenotype with evidence of inflammation, hepatocyte injury and hepatic stellate cell activation not seen in HFD controls or LFD HMGCS2-knockdown mice.

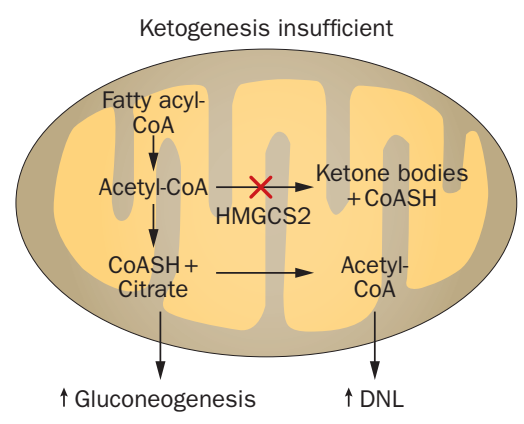

Model of metabolic maladaption to ketogenic insufficiency. Image produced in consultation with P. A. Crawford.

In contrast to ketogenicinsufficient mice on the LFD, those on the HFD had a lower glycaemic status. Metabolomic profiling of ketogenic-

insufficient mice showed that when fatty acids are in excess, coenzyme A (CoASH, a product of ketogenesis) is depleted, which disrupts the tricarboxylic acid (TCA) cycle and reduces gluconeogenesis. DNL remains enhanced as excess acetyl-CoA does not enter the dysfunctional TCA cycle. These findings were supported by higher mRNA expression of factors involved in DNL in ketogenic-insufficient mice than in controls.

In an attempt to rescue glucose production, the investigators perfused livers of HMGCS2knockdown mice with exogenous CoASH precursors (pantothenic acid and cysteine), which normalized the TCA cycle and gluconeogenesis.

Crawford says that future research aims to "test the hypothesis that obesity is a state of ketogenic insufficiency and that selectively enhancing ketogenesis might provide opportunities to mitigate the metabolic consequences of over-nutrition".

Gillian Patman

Original article Cotter, D. G. et al. Ketogenesis prevents diet-induced fatty liver injury and hyperglycemia. J. Clin. Invest. doi:10.1172/ JCI76388 